\title{
COPD AFFECTED LUNG TISSUE REMODELLING DUE TO THE LOCAL DISTRIBUTION OF MMP-2, TIMP-2, TGF- $\beta 1$ AND HSP-70
}

\author{
Zane Vitenberga $^{1}$, Māra Pilmane ${ }^{1}$, Aurika Babjoniševa ${ }^{2}$ \\ ${ }^{1}$ Department of Morphology, Institute of Anatomy and Anthropology, \\ Riga Stradiňš University, Riga, Latvia \\ ${ }^{2}$ Pauls Stradiņš Clinical University Hospital, Riga, Latvia
}

\begin{abstract}
Chronic obstructive pulmonary disease (COPD) is strongly associated with progressive airway limitation where the key mechanism is abnormal airway remodelling of bronchial mucosa maintained by complex crosstalk of tissue remodelling and regulatory factors. The aim of this study was to determine the appearance and local distribution of tissue remodelling factors in COPDaffected lung tissue and to compare the findings with the control group. In this study, lung tissue specimens were obtained from 27 patients with COPD and 49 control patients. Tissue samples were examined by routine hematoxylin and eosin staining. Remodelling and regulatory factors matrix metalloproteinase-2 (MMP-2), tissue inhibitor of metalloproteinase-2 (TIMP-2), transforming growth factor- $\beta 1$ (TGF- $\beta 1$ ), as well as heat shock protein-70 (Hsp-70) were detected by immunohistochemistry in airway mucosa. The numbers of positive structures were evaluated semiquantitatively. Non-parametrical statistical analysis was performed. Overall COPD-affected lung tissue presented chronic inflammation and tissue remodelling in routine histological analysis. Compared to the control group, statistically significant $(\mathrm{P}<0.05)$ difference was calculated between COPD affected lung tissue and control group with overall more immunoreactive cells containing MMP-2, TIMP-2 and TGF- $\beta 1$ and less Hsp-70 immunoreactive bronchial epithelial cells, subepithelial connective tissue fibroblasts, bronchial smooth muscle cells and secretory cells of bronchial glands with more pronounced findings of TGF- $\beta 1$, however, less Hsp-70. Study findings suggest pronounced tissue damage and remodelling with the local regulatory environment of up-regulated TGF- $\beta 1$. Increased numbers of MMP-2, TIMP-2 and TGF- $\beta 1$ immunoreactive cells suggest the
\end{abstract}


key role of these remodelling factors in COPD pathogenesis. Decrease of Hsp-70 proves increased cell damage in COPD-affected airway mucosa.

Keywords: COPD; remodelling; lung; airways; immunohistochemistry

\section{INTRODUCTION}

Chronic obstructive pulmonary disease (COPD) is a chronic inflammatory lung disease that causes obstructed airflow through the lung airways. In COPD, mostly the pathogenic triad of chronic inflammation, altered and disrupted remodelling, as well as oxidative stress correspond to the cytopathology and histopathology [6]. Altered inflammatory response, cell and tissue damage, also abnormal repair of cell and tissue injury confirms defective remodelling of the extracellular matrix (ECM) in lung airways. ECM in airways is composed of matrix proteins, as well as molecular environment modifying molecules like matrix metalloproteinases (MMPs), growth and regulatory factors and signalling molecules [18]. Matrix metalloproteinase 2 (MMP-2) from the gelatinase family strongly affects the ECM components in tissue remodelling, as well as connective tissue cell migration and wound healing that might lead to fibrosis [14]. Tissue inhibitors of metalloproteinases (TIMPs) are acting as direct inhibitors of MMPs; moreover, TIMPs like tissue inhibitor of metalloproteinase 2 (TIMP-2) promote cell growth and protect cells from apoptosis. TIMPs not only affect tissue remodelling, but also shape and organize appropriate ECM environment and architecture. Increased levels of TIMPs promote ECM accumulation towards fibrosis, whereas the decrease of TIMPs enhances proteolysis in ECM. TIMPs regulate MMPs within ECM by inhibiting their maintenance $[1,6,7]$. Overall effective ECM remodelling in lung tissue is regulated as a dynamic balance between the proper cell and tissue renewal and, conversely, the tissue degradation. In COPD, airway remodelling occurs as a resistance response to the cigarette smoking induced damage to airway cells and tissues [16]. Tissue remodelling aspect of COPD pathogenesis in molecular level predominantly contributes by an imbalance of proteolytic and antiproteolytic mechanisms with pathological ECM degradation or controversially, ECM deposition [6]. Extensive production of ECM components with imbalance of MMPs and TIMPs to build remodelled tissue is evaluated as crucial in COPD pathogenesis with activated bronchial epithelium that further triggers subepithelial connective tissue to promote airway fibrosis with rapid 
differentiation of fibroblasts into myofibroblasts $[9,17]$. Remodelling of central airways in COPD due to the increased deposition of ECM proteins, as well as an increase of smooth muscle cell area leads to airway obstruction initiating luminal narrowing [9]. The transforming growth factor $\beta 1$ (TGF- $\beta 1$ ) is the most abundant member of the transforming growth factor (TGF) family in lung tissue. TGF- $\beta 1$ promotes the secretion and deposition of ECM proteins by airway fibroblasts and myofibroblasts $[9,11]$. Secretion of TGF- $\beta 1$ by injured epithelial cells induces airway epithelial wound repair and further subepithelial ECM remodelling [4]. 70-kDa heat shock protein (Hsp-70) is intracellularly located molecular chaperone with regulatory role of extracellular chemokine production influencing both normal innate immunity responses and inflammatory signalling [5]. Synthesis of Hsp-70 is upregulated during different stresses (physical or chemical stress), especially during oxidative stress, thus it maintains protective effects against cell and tissue damage universally in human tissue [13]. Reactive oxygen species (ROS) as oxidative stress stimuli promoted by cigarette smoking could mediate cell and tissue alterations, as well as cell senescence in COPD. ROS also interact with proteases and antiproteases then triggering the imbalance of both. Hsp-70 as oxidative stress biomarker therefore could contribute in COPD pathogenesis within oxidative injury [6].

Although many molecular signalling pathways are studied in COPD pathogenesis, they eventually fail leading to abnormal and altered cell crosstalk, regulation and adaption. Thus, the aim of this study was to explore the appearance and local distribution of tissue remodelling and regulatory factors in COPDaffected lung tissue.

\section{MATERIALS AND METHODS}

COPD-affected lung tissue specimens were obtained during fibrobronchoscopy from 27 patients aged from 53 to 85. The diagnosis of COPD was assessed by both clinical criteria and during bronchoscopy. The lung tissue material of the control group was obtained during autopsy from 49 relatively healthy study subjects aged from 9 to 95 years. The study was approved by the Ethical Committee of Pauls Stradiňš Clinical University Hospital (23 January 2013). Tissue sections $(5 \mu \mathrm{m})$ were cut to proceed to routine histological staining with hematoxylin and eosin. All lung tissue specimens were examined by bright field light microscopy. Tissue remodelling and regulatory markers were studied using the biotin-streptavidin immunohistochemistry (IHC) method. Primary 
antibodies of MMP-2 (R\&D Systems, Germany), TIMP-2 (Santa Cruz Biotechnology, USA), TGF- $\beta 1$ (Biorbyt, UK), and Hsp-70 (Invitrogen, Italy) were used in this study. The appearance and distribution of immunoreactive (positive) cells was evaluated semi-quantitatively [12], counting the relative number of positive epithelial cells, mucosal fibroblasts, glandulocytes, bronchial smooth muscle cells and capillary endothelial cells. The following scale of semi-quantitative method was used, grading the positive cells seen in the visual field at $\times 400$ magnification: 0 - no positive cells, $0 /+-$ occasional, +- few,$+/++-$ few to moderate number, ++- moderate number, $++/+++-$ moderate number to numerous, +++- numerous, $+++/++++-$ numerous to abundance, ++++abundance of positive cells. To perform statistical analysis, nonparametric statistical methods - Mann-Whitney U test and Spearman's rank-order correlation - were used. All data were categorized in ordinal ranking as ordinal values, where the ranking system of nine ranks was applied from the value of 0 (no positive structures (0)), the value 0.5 (occasional positive cells $(0 /+)$ ), et cetera to the value of 4.0 (abundance $(++++)$ ).

\section{RESULTS}

In routine histological analysis, COPD-affected lung tissue presented various degrees of chronic inflammation and tissue remodelling. We evidenced goblet cell hyperplasia (Fig. 1A), bronchial epithelial squamous metaplasia, subepithelial basement membrane thickening, airway fibrosis with thick connective tissue collagen bundles, bronchial gland hypertrophy and hyperplasia (Fig. 1B), bronchial microvasculature remodelling, smooth muscle cell hyperplasia and hypertrophy, remarkable inflammatory cell infiltration in airway mucosa and submucosa, and glandular tissue.

The numbers of MMP-2 positive cells varied from no positive cells (0) in the connective tissue, bronchial glands, smooth muscles and in the endothelium to abundance $(++++)$ of positive cells in the bronchial epithelium (Table 1). Overall, the appearance of MMP-2 immunoreactive cells was evaluated as highest in the bronchial epithelium and connective tissue, however, we found fewer MMP-2 positive cells in the bronchial smooth muscle. The numbers of TIMP-2 immunoreactive cells were estimated ranging from no positive cells (0) in the bronchial glands reaching the maximum value of abundance $(++++)$ of cells in the bronchial epithelium and from numerous to abundance $(+++/++++)$ of TIMP-2-containing cells in the connective tissue and 

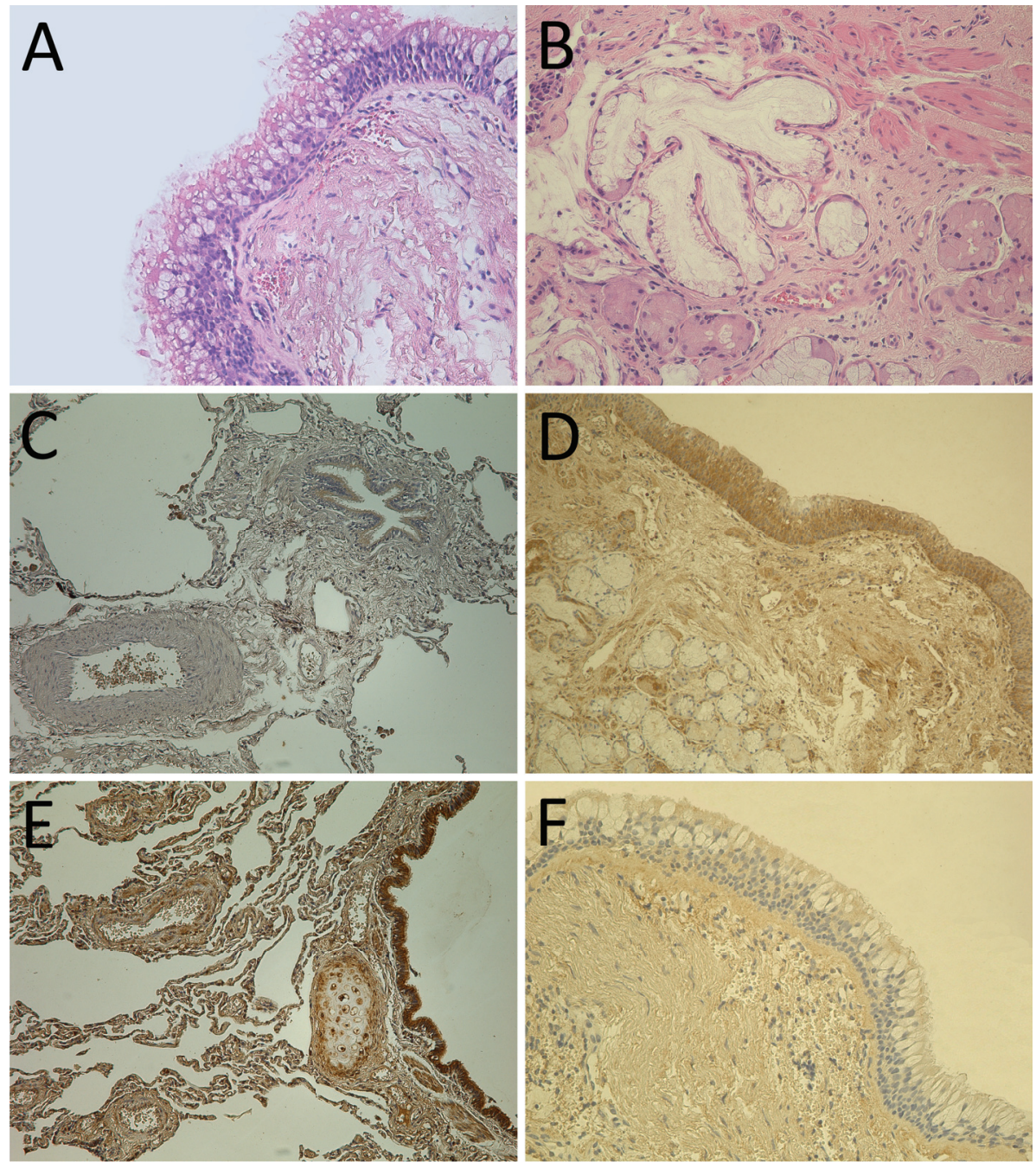

Figure1. Representative microphotographies examined by routine histological staining with hematoxylin and eosin (A, B) and detection of remodelling factors MMP-2 and $\mathrm{Hsp}-70$ by use of the immunohistochemistry (IMH) method (C - F). (A) Goblet cell hyperplasia of bronchial epithelium in bronchus wall of a 64-year-old male (COPD). Hematoxylin and eosin, X200. (B) Bronchial gland hypertrophy and hyperplasia, smooth muscle hypertrophy in large bronchus wall of a 60-year-old-male (COPD). Hematoxylin and eosin, X200. (C) Few to moderate number (+/++) of MMP-2 positive bronchial epithelial cells of large bronchus wall in an 81-year-old female (control). MMP-2 IMH, X100. (D) Numerous to abundance $(+++/++++)$ of MMP-2 positive epithelial cells in bronchial epithelium with squamous epithelial metaplasia in a 60-year-old male (COPD). MMP-2 IMH, 100x. (E) Abundance $(++++)$ of $\mathrm{Hsp}-70$ immunoreactive bronchial epithelial cells in bronchus wall of an 81-yearold female (control). Hsp-70 IMH, X100. (F) Occasional (0/+) Hsp-70 positive bronchial epithelial cells in bronchus wall of a 72-year-old male (COPD). Hsp-70 IMH, X200. 


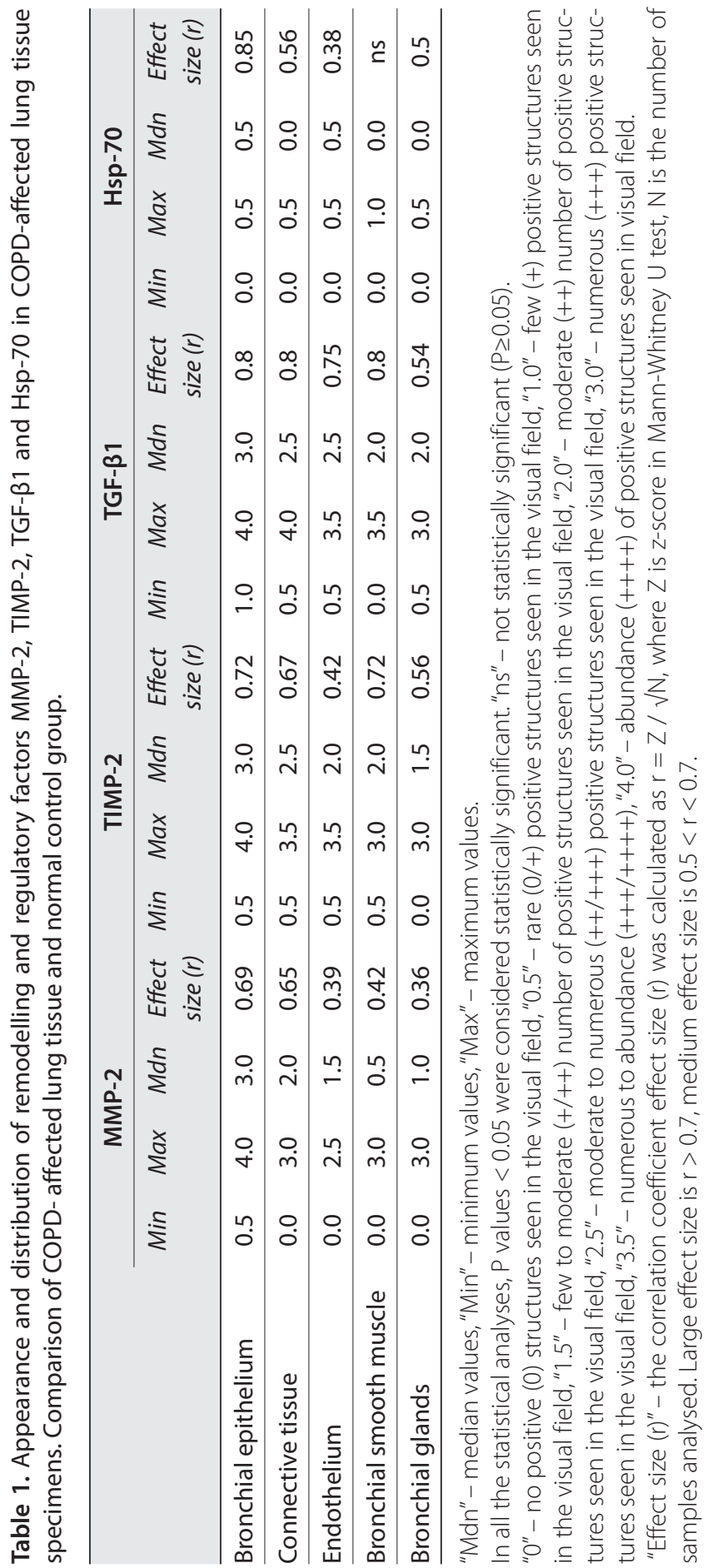


the endothelium. In summary, we found the most of TIMP-2 immunoreactive cells in the bronchial epithelium and fewer in the bronchial smooth muscle. Moreover, immunohistochemistry findings of TIMP-2 were more pronounced when compared to MMP-2 results in all the examined tissues. The appearance and distribution of TGF- $\beta 1$ varied from no positive (0) TGF- $\beta 1$ containing smooth muscle cells to abundance ( ++++$)$ of TGF- $\beta 1$ containing bronchial epithelial cells and subepithelial connective tissue fibroblasts. Overall, the higher numbers of TGF- $\beta 1$ immunoreactive cells were found in the bronchial epithelium and connective tissue and somewhat fewer in the bronchial glands, endothelium and among the bronchial smooth muscle cells. In contrast to MMP-2, TIMP-2, TGF- $\beta 1$, we found particularly few Hsp-70-containing immunoreactive cells with the range of no positive (0) cells in all the examined tissues to few (+) Hsp-70 positive bronchial smooth muscle cells. Interestingly, we found overall more pronounced numbers of bronchial epithelial cells and fewer Hsp-70 positive endothelial cells with the estimation of mostly occasional $(0 /+)$ numbers of cells.

Compared to the control group, Mann-Whitney U-test showed a statistically significant $(\mathrm{P}<0.05)$ difference between COPD-affected lung tissue and the control group with overall more immunoreactive cells in all the examined tissues of bronchial epithelium, subepithelial connective tissue, bronchial glands, small capillary endothelium and bronchial smooth muscle containing more MMP-2 (Fig. 1C, Fig. 1D), TIMP-2 and TGF- $\beta 1$ and fewer Hsp-70 (Fig. 1E, Fig. 1F) immunoreactive cells. With large effect size $r$ calculated $(>0.7)$ to specify Mann-Whitney U test results, COPD-affected lung tissue is more likely to evidence an increased number of MMP-2, TIMP- 2 and TGF- $\beta 1$ immunoreactive cells. Bronchial epithelial cells, subepithelial connective tissue fibroblasts, bronchial smooth muscle cells and endothelial cells indicated more pronounced findings of TGF- $\beta 1$, however, fewer Hsp-70 immunoreactive bronchial epithelial cells compared to the normal control group (Table 1).

\section{DISCUSSION}

In COPD-affected lung tissue, MMP-2 immunoreactive cells were ranked higher in bronchial epithelium and subepithelial connective tissue (median values 3.0 and 2.0, respectively). These findings suggest COPD tissue is more likely to evidence an increase of MMP-2 in these compartments of the bronchial mucosa compared to the healthy control group. In COPD patients with 
the background of cigarette smoking, air-derived irritants might harm the epithelial tissue and initiate inflammation with attracting immune cells and activating proteolytic environment with the predominance of collagenase MMP-2. Furthermore, degraded extracellular matrix (ECM) fragments might act as local chemokines and promote local inflammation [2]. MMP-2 gene expression is induced by cigarette smoke in normal lung connective tissue fibroblasts [17]. Also in COPD-affected lung tissue, lung fibroblasts show induced MMP-2 protein expression, as well as increased MMP-2 gelatinase activity against ECM [10]. Immunoreactivity, protein expression, gelatinolytic activities of MMP-2 was markedly increased in epithelial cells, fibroblasts and endothelial cells in COPD suggesting up-regulation of MMP-2 [8]. Also, when compared to the control group, the medium effect size $(0.5<r<0.7)$ was calculated for MMP-2 immunoreactive bronchial epithelial cell and connective tissue fibroblasts suggesting their increased role in COPD development.

Similarly to the findings of MMP-2, we found more pronounced TIMP-2 appearance in bronchial epithelium and connective tissue; moreover, high numbers of immunoreactive TIMP-2 endothelial cells were also found (median values 3.0, 2.5 and 2.0, respectively). To maintain the stability of ECM environment, TIMP-2 directly inhibits MMP-2; therefore, the location of TIMP-2 is crucial. After the release, both active form and pro-active form of MMPs are regulated by the activity of TIMPs [1]. In COPD, imbalance of proteases (MMPs) and antiproteases (TIMPs) induces structural changes in the form of pathological tissue repair and airway remodelling [17]. In our study, we might support this opinion of TIMP-2 predominance over MMP-2 with its regulatory role in airway remodelling. Moreover, these findings might suggest the background of airway fibrosis that we estimated as ECM depositions with thick collagen fibre bundles in our patients.

Compared to all other examined remodelling and regulatory factors, we counted more TGF- $\beta 1$ immunoreactive cells in all the examined tissue compartments (median values were ranging from 2.0 to 3.0) of COPD-affected bronchial mucosa with more pronounced findings in the bronchial epithelium. When stimulated with TGF- $\beta 1$, airway basal epithelial cells might lead the whole bronchial epithelium to squamous metaplasia and goblet cell hyperplasia or contribute to airway fibrosis through epithelial to mesenchymal transition [15]. The background then of fibrosis in airways is master regulation of TGF- $\beta 1$ with the consequences of pseudostratified epithelial to mesenchymal transition, as well as fibroblast to myofibroblast and even smooth muscle cell 
to myofibroblast transition [9]. TGF- $\beta 1$ release based on oxidant-mediated mechanisms confirms the activating role of oxidative stress $[3,9]$. In our study, the most pronounced site of TGF- $\beta 1$ was exactly bronchial epithelium. Since the epithelium is affected by cigarette smoke first, epithelial cells must tolerate oxidative injury with an increased release of regulatory molecules suggesting their protective role. Airway epithelium, activated smooth muscle, fibroblasts and myofibroblasts are important sources of TGF- $\beta 1$, moreover, with a powerful effect on tissue remodelling [7]. TGF- $\beta 1$ exerts a protective role by struggling to maintain lung tissue homeostasis in physiological conditions that are usually lost in COPD [3].

In all the examined tissue compartments, we found fewer or similar numbers of Hsp-70 immunoreactive cells overall and compared to the control group. The examined cells were mostly negative (0) for Hsp-70 immunostaining, however, reaching only occasional (median value 0.5 ) numbers of bronchial epithelial cells that had the highest values in the COPD group. In the control tissue, the numbers of Hsp-70 immunoreactive cells were few to moderate (median value 1.5) in the bronchial epithelium and occasional (median value 0.5 ) in the bronchial connective tissue and bronchial glands. Although Hsp-70 maintains the protective role against cytotoxic damage and it is up-regulated under physical and chemical stress stimuli, abnormal Hsp-70 expression might occur if prolonged stress stimuli keep cellular and tissue stress response. When chronic cigarette smoking occurs, long-term oxidative stress might cause oxidative damage due to accumulation of oxidative products in airway tissue [13]. Adaption to chronic oxidative stress occurs through down-regulation of Hsp-70. Moreover, short-time exposure of cigarette smoke initiates an increased expression of Hsp-70. Conversely, long-term exposure caused dramatically decreased expression of $\mathrm{Hsp}-70$, genotoxicity altogether with DNA damage, cell and tissue injury [19].

In conclusion, the wide distribution and extensive appearance of MMP-2, TIMP-2, TGF- $\beta 1$, and Hsp-70 proposes pronounced tissue damage and remodelling with the local regulatory patterns of increased TGF- $\beta 1$. Increased numbers of MMP-2, TIMP- 2 and TGF- $\beta 1$ immunoreactive cells suggest the key role of these remodelling factors in COPD pathogenesis. Decrease of Hsp-70 proves its impaired function and increased cell damage in COPD affected lung tissue. 


\section{REFERENCES}

1. Arpino V., Brock M., Gill S.E. (2015). The role of TIMPs in regulation of extracellular matrix proteolysis. Matrix Biol, 44-46, 247-254.

https://doi.org/10.1016/j.matbio.2015.03.005

2. Bagdonas E., Raudoniute J., Bruzauskaite I., Aldonyte R. (2015). Novel aspects of pathogenesis and regeneration mechanisms in COPD. Int J Chron Obstruct Pulmon Dis, 10, 995-1013.

3. Baraldo S., Bazzan E., Turato G., Calabrese F., Beghé B., Papi A., Maestrelli P., Fabbri L.M., Zuin R., Saetta M. (2005). Decreased expression of TGF-beta type II receptor in bronchial glands of smokers with COPD. Thorax, 60, 12, 998-1002. https://doi.org/10.1136/thx.2005.046292

4. Boxall C., Holgate S.T., Davies D.E. (2006). The contribution of TGF-beta and epidermal growth factor signalling to airway remodelling in chronic asthma. Eur Respir J, 27, 1, 208-229. https://doi.org/10.1183/09031936.06.00130004

5. Dong J., Guo L., Liao Z., Zhang M., Zhang M., Wang T., Chen L., Xu D., Feng Y., Wen F. (2013). Increased expression of heat shock protein 70 in chronic obstructive pulmonary disease. Int Immunopharmacol, 17, 3, 885-893. https://doi.org/10.1016/j.intimp.2013.09.003

6. Fischer B.M., Pavlisko E., Voynow J.A. (2011). Pathogenic triad in COPD: oxidative stress, protease-antiprotease imbalance, and inflammation. Int J Chron Obstruct Pulmon Dis, 6, 413-421. https://doi.org/10.2147/COPD.S10770

7. Ghanei M., Ghalejooghi N.A., Nourani M.R., Harandi A.A., Fooladi A.A. (2010). Effect of TGFß1 and TIMP2 on disease activity in asthma and COPD. Iran J Allergy Asthma Immunol, 9, 2, 79-86.

8. Li H., Cui D., Tong X., Ma N., Gao Y., Cui X., Lu L., Wang D., Liang Y. (2002). The role of matrix metalloproteinases in extracellular matrix remodelling in chronic obstructive pulmonary disease rat models. Zhonghua Nei Ke Za Zhi, 41, 6, 393-398.

9. Milara J., Peiró T., Serrano A., Cortijo J. (2013). Authors' response to: epithelial mesenchymal transition (EMT) in small airways of COPD patient. Thorax, 68, 8, 784. https://doi.org/10.1136/thoraxjnl-2013-203484

10. Ning W., Dong Y., Sun J., Li C., Matthay M.A., Feghali-Bostwick C.A., Choi A.M. (2007). Cigarette smoke stimulates matrix metalloproteinase-2 activity via EGR-1 in human lung fibroblasts. Am J Respir Cell Mol Biol, 36, 4, 480-490. https://doi.org/10.1165/rcmb.2006-0106OC

11. Pegorier S., Campbell G.A., Kay A.B., Lloyd C.M. (2010). Bone Morphogenetic Protein (BMP)-4 and BMP-7 regulate differentially Transforming Growth Factor (TGF)- $\beta 1$ in normal human lung fibroblasts. Respir Res, 11, 1, 85. https://doi.org/10.1186/1465-9921-11-85

12. Pilmane M., Rumba I., Sundler F., Luts A. (1998). Patterns of occurrence and distribution of neuroendrocine elements in lungs of humans with chronic lung 
diseases. Proceedings of the Latvian Academy of Sciences Section B Natural Exact and Applied Sciences, 52, 3-4, 144-152.

13. Qu B., Jia Y., Liu Y., Wang H., Ren G., Wang H. (2015). The detection and role of heat shock protein 70 in various nondisease conditions and disease conditions: a literature review. Cell Stress \& Chaperones, 20, 6, 885-892. https://doi.org/10.1007/s12192-015-0618-8

14. Rohani M.G., Parks W.C. (2015). Matrix remodeling by MMPs during wound repair. Matrix Biol, 44-46, 113-121. https://doi.org/10.1016/j.matbio.2015.03.002

15. Shaykhiev R., Crystal R.G. (2014). Early events in the pathogenesis of chronic obstructive pulmonary disease. Smoking-induced reprogramming of airway epithelial basal progenitor cells. Ann Am Thorac Soc, 11, Suppl 5, 252-258. https://doi.org/10.1513/AnnalsATS.201402-049AW

16. Sohal S.S., Ward C., Walters E.H. (2014). Importance of epithelial mesenchymal transition (EMT) in COPD and asthma. Thorax, 69, 8, 768. https://doi.org/10.1136/thoraxjnl-2014-205582

17. Srivastava P.K., Dastidar S.G., Ray A. (2007). Chronic obstructive pulmonary disease: role of matrix metalloproteases and future challenges of drug therapy. Expert Opin Investig Drugs, 16, 7, 1069-1078. https://doi.org/10.1517/13543784.16.7.1069

18. White E.S. (2015). Lung Extracellular Matrix and Fibroblast Function. Ann Am Thorac Soc, 12, Suppl 1, 30-33. https://doi.org/10.1513/AnnalsATS.201406-240MG

19. Wu X.J., Luo G.X., Zeng X., Lan L.L., Ning Q., Xu Y.J., Zhao J.P., Xie J.G. (2013). Genotoxicity and reduced heat shock protein 70 in human airway smooth muscle cells exposed to cigarette smoke extract. J Huazhong Univ Sci Technolog Med Sci, 33, 6, 827-833. https://doi.org/10.1007/s11596-013-1206-8

\section{Address for correspondence:}

Zane Vitenberga

Institute of Anatomy and Anthropology, Department of Morphology

Riga Stradiņš University

Kronvalda Boulevard 9, Riga, LV-1010, Latvia

Phone: +37167320862

E-mail: Zane.Vitenberga@rsu.lv 